\title{
PROFISSIONAIS DE ENFERMAGEM E A INTERNAÇÃO PSIQUIÁTRICA EM HOSPITAL GERAL: PERCEPÇÕES E CAPACITAÇÃO PROFISSIONAL
}

\author{
Marciana Fernandes Moll ${ }^{1}$, Lucas Duarte Silva ${ }^{2}$, Felipe Henrique de Lima Magalhães ${ }^{3}$, Carla Aparecida Arena \\ Ventura $^{4}$
}

RESUMO: Objetivando verificar como a equipe de enfermagem percebe a internação psiquiátrica no hospital geral e identificar sua capacitação profissional para lidar com a pessoa com transtorno mental e/ou que fazem uso prejudicial de álcool e outras drogas, desenvolveu-se este estudo qualitativo. A coleta de dados ocorreu nos meses de maio a julho de 2016, em hospital geral do estado de Minas Gerais e participaram 31 profissionais de enfermagem. Utilizou-se a entrevista semiestruturada para a obtenção dos dados que foram submetidos à análise de conteúdo e análise temática. Verificou-se que os participantes têm dificuldades em aceitar a internação psiquiátrica no hospital geral, o que se relaciona à possível inaptidão para oferecer cuidados direcionados à pessoa com transtorno mental. Sendo assim, faz-se necessário o desenvolvimento de planejamento educativo, sobretudo para os técnicos de enfermagem, com vistas a qualificá-los para cuidar dessas pessoas.

DESCRITORES: Enfermagem; Psiquiatria; Hospitalização; Percepção; Capacitação.

\section{NURSING PROFESSIONALS AND PSYCHIATRIC ADMISSION IN GENERAL HOSPITAL: PERCEPTIONS AND PROFESSIONAL TRAINING}

\begin{abstract}
Qualitative study aimed to investigate how the nursing staff perceives psychiatric care beds in a general hospital and assess whether they are capable toassist people with mental disorders. Data was collected from May to July 2016, in a general hospital in the state of Minas Gerais and 31 nurses participated in the study. Semi-structured interviews were used to collect information, and the data obtained was subsequently submitted to content and thematic analysis. It was found that the participants felt uncomfortable withpsychiatric beds in the general hospital, which may be related to their lack of skills to provide care for people with mental disorders. Therefore, it is necessary to develop educational planning, especially for nursing technicians, in order to make them able to assist these patients.

DESCRIPTORS: Nursing; Psychiatry; Hospitalization; Perception; Training.
\end{abstract}

\section{PROFESIONALES DE ENFERMERÍA Y LA INTERNACIÓN PSIQUIÁTRICA EN HOSPITAL GENERAL: PERCEPCIONES Y CAPACITACIÓN PROFESIONAL}

RESUMEN: Se objetivó verificar como es percibida la internación psiquiátrica en hospital general por parte del equipo de enfermería, y verificar su capacitación para encargarse de personas con trastorno mental y/o que hacen uso perjudicial de alcohol y otras drogas. Estudio cualitativo cuyos datos fueron recolectados de mayo a julio de 2016, en hospital general de Minas Gerais, participaron 31 profesionales de enfermería. Se aplicó entrevista semiestructurada para obtención de los datos, sometidos a análisis de contenido y análisis temático. Se verificó que los participantes tenían dificultades para aceptar la internación psiquiátrica en el hospital general lo cual está relacionado con la posible ineptitud para brindar cuidados dirigidos a personas con trastorno mental. Siendo así, resulta necesario el desarrollo de planificación educativa, particularmente para los técnicos de enfermería, a fin de calificarlos para brindar cuidados a estas personas.

DESCRIPTORES: Enfermería; Psiquiatría; Hospitalización; Percepción; Capacitación.

${ }^{1}$ Enfermeira. Doutora em Ciências. Docente de Graduação e Pós-graduação em Enfermagem na Universidade de Uberaba. Uberaba, MG, Brasil.

${ }^{2}$ Enfermeiro. Mestrando em Enfermagem Psiquiátrica. Universidade de São Paulo. Ribeirão Preto, SP, Brasil.

${ }^{3}$ Discente de Enfermagem. Universidade de Uberaba. Uberaba, MG, Brasil.

${ }^{4}$ Livre Docente. Docente da Universidade de São Paulo. Ribeirão Preto, SP, Brasil.

Autor Correspondente:

Marciana Fernandes Moll

Universidade de Uberaba

Av. Nenê Sabino, 1801 - 38055-500 - Uberaba, MG, Brasil

E-mail:mrcna13@yahoo.com.br
Recebido: 25/12/2016

Finalizado: 25/05/2017 


\section{INTRODUÇÃO}

Inicialmente, o hospital apresentava natureza eminentemente religiosa e era tido como ambiente de caridade, onde os enfermos recebiam apenas cuidados de caráter social e espiritual ${ }^{(1)}$. Entretanto, com os avanços das práticas em saúde e dos métodos de assistência, pessoas de classes sociais mais elevadas passaram a procurar os serviços hospitalares, que de abrigos se transformaram em locais disponibilizados para cuidados científicos e assistenciais ${ }^{(2)}$.

O hospital pode abranger várias especialidades, classificadas pelo tipo de serviço de saúde oferecido, podendo ser assistência especializada ou geral ${ }^{(3)}$. Leitos psiquiátricos começaram a ser implantados em Hospitais Gerais no Brasil em 1960, quando ainda predominava o modelo manicomial de tratamento dos transtornos mentais ${ }^{(4)}$. O foco passou a não se centrar apenas em patologias físicas, reconhecendose a existência de transtornos mentais como problema de natureza social, comportamental e familiar, influenciando a qualidade de vida das pessoas envolvidas.

Contudo, até o movimento de reforma psiquiátrica instituído no Brasil, as pessoas com transtornos mentais continuaram isoladas da sociedade e tratadas em hospitais psiquiátricos. Este movimento fortaleceu-se com a promulgação da Lei 10.216 de 2001, que propulsionou um conjunto legislativo posterior buscando assegurar o respeito aos direitos das pessoas com transtornos mentais, assim como a conformação de uma rede extra-hospitalar que assegurasse a reabilitação psicossocial e maior integração à sociedade ${ }^{(5)}$.

Recentemente, a partir da publicação da Portaria GM/MS n 148, de 31 de janeiro de 2012, os hospitais que comportam leitos psiquiátricos podem ser designados como Serviços Hospitalares de Referência, destinados às pessoas com sofrimento psíquico/transtorno mental e que apresentam necessidades de saúde advindas do uso de álcool, crack e outras substâncias psicoativas ${ }^{(5)}$. Esta modalidade de internação em hospitais gerais traz benefícios que vão além do tratamento oferecido, uma vez que também contribui para a redução do estigma existente sobre a pessoa com transtorno mental ${ }^{(6)}$.

Nesse cenário, dados de pesquisa realizada em 2010 corroboraram a necessidade de mudanças no reconhecimento do transtorno mental e seu tratamento, informando que hospitais gerais recebiam grande quantidade de pessoas com algum tipo de sofrimento mental. A morbidade psiquiátrica desses pacientes variava de $20 \%$ a $60 \%$ e, dessa parcela, somente $1 \%$ a $13 \%$ passavam por atendimento

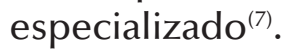

A internação de pessoas com transtorno mental em hospitais gerais pode ocorrer em situações específicas, como o desenvolvimento de patologia clínica associada à doença mental já existente ou a manifestação de um surto durante hospitalização clínica ${ }^{(8)}$.

De forma mais específica, estudo evidenciou que aproximadamente $30 \%$ dos pacientes internados em unidades de clínica médica e cirúrgica, para o tratamento de problemas clínicos, apresentavam alguma doença psiquiátrica. Os diagnósticos psiquiátricos mais prevalentes foram: transtornos de humor (35\%); transtornos de ansiedade (20\%); transtornos mentais devido ao abuso/uso de substâncias $(20 \%)$; transtornos mentais orgânicos (20\%); e outros tipos de transtornos mentais $(5 \%)^{(9)}$.

Desse modo, a equipe de enfermagem atuante nos hospitais gerais, especificamente em unidades de internação psiquiátrica ou em unidades com leitos psiquiátricos, deve se sensibilizar para compreender e suprir as necessidades das pessoas com transtorno mental em regime de internação.

Para tanto, é fundamental que a atuação da enfermagem ultrapasse os procedimentos técnicos e estabeleça também intervenções promotoras do bem-estar psíquico, visando assegurar a saúde do ser humano. Desse modo, este artigo apresenta como objetivos: verificar como a equipe de enfermagem percebe a internação psiquiátrica no hospital geral, e identificar sua capacitação profissional para lidar com a pessoa com transtorno mental e/ou que fazem uso prejudicial de álcool e outras drogas. Este conhecimento é importante para caracterizar a enfermagem no processo de internação psiquiátrica em hospital geral.

\section{- METODOLOGIA}


Estudo descritivo de abordagem qualitativa desenvolvido em uma cidade com aproximadamente 300 mil habitantes, localizada no interior de Minas Gerais. O hospital geral que constituiu o cenário da investigação possuía 225 leitos, dos quais seis se destinam às pessoas com transtornos mentais e/ou que faziam uso prejudicial de álcool e outras drogas.

Todos os profissionais de Enfermagem que atuavam na clínica médica com leitos psiquiátricos foram convidados a participar desta investigação, totalizando 33 profissionais. Destes, 31 aceitaram participar: 28 (90,32\%) mulheres, $27(87,09 \%)$ casados e quatro (3,22\%) solteiros. Foram 26 técnicos de Enfermagem (83,87\%) e cinco Enfermeiros (16,12\%).

Os dados foram coletados nos meses de maio a julho de 2016, por meio da entrevista semiestruturada. As questões envolviam os seguintes aspectos: percepção do profissional sobre a internação psiquiátrica no hospital geral e capacitação profissional para lidar e interagir com pessoas internadas por problemas psiquiátricos no hospital geral.

Antes do início da coleta dos dados, um dos pesquisadores abordava o profissional de enfermagem e o convidava a participar do estudo. Depois da aceitação, era agendada a entrevista em um local reservado no próprio hospital, estipulado pelo próprio participante. Tais entrevistas foram gravadas e posteriormente transcritas.

Os dados foram analisados por meio de análise de conteúdo, que constitui um conjunto de técnicas de análise de comunicação, visando a sistematização do conteúdo das mensagens, efetuando deduções lógicas e justificadas, considerando emissor, contexto e efeitos ${ }^{(10)}$.

Após essa técnica de análise, os dados foram sistematizados e analisados, também por meio da análise temática, na qual foram identificados conteúdos que convergiram para significados semelhantes e foram agrupados em unidades temáticas ${ }^{(11)}$. Este método de análise se fundamentou nas diretrizes que estabelecem as seguintes fases: pré-análise, exploração do material e tratamento dos resultados por meio da inferência e da interpretação ${ }^{(10)}$.

Iniciou-se a investigação após a aprovação do Comitê de Ética (Parecer no 447.029) emitido após análise do Comitê de Ética em Pesquisas da Universidade de Uberaba. Posteriormente, todos os participantes receberam esclarecimentos sobre os objetivos e assinaram o Termo de Consentimento Livre e Esclarecido. A fim de proteger o anonimato dos participantes, todos foram identificados pelas letras TE (Técnicos de Enfermagem) com números sequenciais e E (Enfermeiros) com números sequenciais.

\section{- RESULTADOS}

Após transcrição, da análise dos dados emergiram dois temas: "Aceitação e sentimentos da equipe de Enfermagem quanto à internação psiquiátrica no hospital geral" e "Capacitação e preparação da equipe de Enfermagem diante das necessidades das pessoas com transtorno mental".

\section{Aceitação e sentimentos da equipe de Enfermagem quanto à internação psiquiátrica no hospital geral}

Identificou-se que prevalece a dificuldade de aceitação dos profissionais de enfermagem frente à internação psiquiátrica no hospital geral:

Tivemos que aceitar e tudo começou da noite para o dia, mas é difícil porque já temos muito trabalho. (E4)

É difícil e são poucos os técnicos que querem cuidar deles. (E5)

Acho que deveria ser um setor específico para eles, porque eu acho que corre o risco tanto a gente que trabalha, quanto os outros pacientes normais internados. (TE23)

Entretanto, houve relatos de alguns participantes, especialmente enfermeiros, que referiram tranquilidade e bem-estar ao cuidarem de pessoas com transtornos psiquiátricos. 
É um tipo de paciente que eu adoro lidar. Me sinto tranquilo. (E1)

É tranquilo, porque sempre ficam acompanhados. (E3)

Detectou-se que os profissionais que não se sentem bem com a internação psiquiátrica em hospital geral, justificam suas opiniões por meio de sentimentos como medo, dificuldade de identificação e insegurança.

Tenho medo deles. (TE9)

Não me identifico com esse tipo de paciente, de jeito nenhum. (TE8)

Me faz sentir muito medo, principalmente quando todos estão dormindo. Eu tenho medo de me agredir. (TE21)

Me sinto insatisfeita com a presença deles aqui, principalmente porque é muito perto da maternidade (E2)

Apesar de alguns membros da equipe mencionarem insegurança ao lidar com pessoas com transtornos mentais, é interessante notar um sentimento generalizado de preocupação com seu bemestar enquanto permanecem em regime de internação:

É preciso orientar a equipe, pois ainda há muito preconceito [...]. As enfermarias são separadas, porém o local não é adequado: aqui tudo pode ser uma arma pra ele se machucar, quebrar alguma coisa. Não vejo problemas em misturar a Clínica Médica com Psiquiatria, porém o local deveria ser mais preparado. (E1)

Foi possível verificar a existência de ideias prévias relacionadas à agressividade e imprevisibilidade das pessoas com transtornos mentais, que persistem mesmo com o uso correto da terapêutica farmacológica, prática prevista durante as hospitalizações.

\section{Capacitação da equipe de enfermagem diante das necessidades das pessoas com transtorno mental}

Neste estudo predominaram os profissionais que referiam não estarem aptos para lidar com as pessoas com transtornos mentais por não terem experiência.

Então, eu nunca vivenciei uma situação de transtorno, mas não pode apavorar, não pode ficar nervoso. (TE20)

Estou aprendendo a lidar com eles na prática. (TE10)

A falta de preparação relatada por alguns participantes foi justificada pela insuficiência qualitativa e quantitativa de capacitação oferecida pelo hospital.

Aquele treinamento foi tão rápido que quando cheguei lá já tinha acabado e depois os pacientes já começaram a ser internados e nunca mais aconteceu nenhum treinamento. (TE16)

Eu aprendi a amarrar na cama em um treinamento rápido que teve quando iam começar as internações. (TE6)

É possível inferir que este treinamento não ofereceu conhecimentos suficientes para que os técnicos de enfermagem oferecessem assistência, centrada na singularidade das necessidades da pessoa que era hospitalizada por intercorrências psiquiátricas.

Em outro aspecto, o cuidado oferecido pelos técnicos de enfermagem que participaram dessa investigação não valoriza as necessidades psíquicas dos sujeitos, uma vez que não diferencia os pacientes com ou sem transtorno mental, tal como foi expresso nos relatos que se seguem.

Vejo que este é um paciente como qualquer outro. (TE13)

É dado o mesmo cuidado que os outros pacientes recebem. (TE2)

Portanto, nota-se que a experiência é um ponto positivo para estabelecer estratégias de cuidado. 
Outrossim, deve-se levar em consideração que nem todos os profissionais tinham experiência para prestarem cuidados em saúde mental ou foram devidamente capacitados para tanto.

De outro modo, alguns dos participantes referiram preparo para atender às demandas de pessoas com transtornos psiquiátricos, o que advém de formações e contatos prévios que ocorreram em outros locais de trabalho.

Porque eu já fiz estágios e já trabalhei um pouco com pacientes psiquiátricos. Então pra mim é natural eu não me assusto com esse tipo de setor. (TE 21)

Desse modo, é necessário que ocorra um planejamento educativo para os técnicos de enfermagem, com vistas a qualificá-los para a identificação das necessidades específicas de pessoas com transtornos mentais internadas em hospitais gerais. A ênfase desta intervenção para os técnicos se deve ao fato deles terem referido nos relatos supracitados, por várias vezes, essa necessidade, o que não foi abordado pelos enfermeiros.

\section{DISCUSSÃO}

Estudos demonstram que, dentre os sentimentos dos profissionais de enfermagem diante de pessoas com transtornos mentais, predominam o medo, a frustração e a impotência ${ }^{(12)}$. Tais sentimentos advêm do estigma histórico atribuído a este sujeito, de ser alguém imprevisível e de difícil socialização ${ }^{(13)}$.

É preciso que a equipe de Enfermagem esteja atenta ao modo como seus sentimentos e opiniões são transmitidos, uma vez que a maneira como a pessoa com transtorno mental é vista e tratada interfere em suas atitudes. Nesse sentido, destaca-se que, ao perceberem que o familiar ou cuidador o olham com certo preconceito, seu comportamento se modifica ${ }^{(14)}$.

Os achados deste estudo são coerentes com estes dados da literatura, ao evidenciarem que 19 profissionais entrevistados apontaram sentimentos negativos como medo, falta de identificação e insegurança. Ao corroborar com o que foi exposto nas falas dos entrevistados, a literatura também aponta que, dentre as principais preocupações associadas à internação psiquiátrica em hospitais gerais, está a inadequação da estrutura física ${ }^{(4)}$.

O ambiente físico e os recursos existentes podem ser propulsores de riscos, tanto para o próprio sujeito como para terceiros em diferentes serviços de saúde, independentemente de ser especializado ou não.

Diante dessa realidade, cabe ao profissional responsável pela prestação do cuidado estar atento a tais recursos e à condição psíquica da pessoa hospitalizada, para adotar métodos de prevenção que evitem auto ou hetero agressão. Ainda é importante acrescentar condutas humanizadas e promotoras de saúde mental, que viabilizem o bem-estar tanto do indivíduo internado, quanto das demais pessoas que estão ao seu redor no ambiente.

Nesse sentido, enfatiza-se que a implantação de leitos psiquiátricos em hospitais gerais configura um serviço relativamente novo na rede de atenção psicossocial, o que pode justificar a pouca aceitação por parte de alguns profissionais. Estes leitos constituem recurso complementar, para oferecer assistência integral a pessoas com transtornos mentais graves que estejam em estado de crise ou que apresentem sintomas agudos, auxiliando a redução do paradigma da discriminação ${ }^{(4,15)}$. Por outro lado, se não forem utilizadas estratégias eficazes e se os profissionais não estiverem preparados, os resultados podem ser extremamente negativos, reforçando o estigma existente na sociedade.

Acredita-se que, por integrar uma equipe multiprofissional, o enfermeiro deve valorizar aspectos biológicos, psíquicos e espirituais das pessoas com transtornos mentais hospitalizadas, considerandoas em sua integralidade, oferecendo escuta ativa que transmita empatia e compreensão. Em caso de não se priorizar essa necessidade, podem acontecer prejuízos nos comportamentos dos pacientes hospitalizados $^{(16)}$.

Desse modo, espera-se que o enfermeiro esteja apto a realizar ações de educação permanente e treinamentos para a equipe de Enfermagem, o que permite abordar técnicas inovadoras e também 
trabalhar a partir das concepções e valores da profissão. Sendo assim, no contexto da equipe de enfermagem, é relevante que o enfermeiro esteja atento e trabalhe com sua equipe esta formação, que pode e deve ocorrer com o apoio das diferentes instâncias do hospital.

O despreparo pode ser também originado da falta de oportunidades de lidar com tais pacientes anteriormente $^{(17)}$. Nesse sentido, verificou-se neste estudo que o despreparo, relatado por alguns participantes, estava associado à pouca ou nenhuma experiência anterior com a assistência às pessoas com transtornos mentais.

Na saúde mental, é fundamental que a equipe de enfermagem busque atualizar-se sobre propostas terapêuticas estabelecidas pela legislação atual (ênfase na inclusão social e reabilitação psicossocial). A capacitação adequada e continuidade de treinamentos são essenciais para elaboração de um plano terapêutico que vise à reinserção social ${ }^{(18)}$.

Os achados desta pesquisa são coerentes com resultados de pesquisas semelhantes encontradas na literatura, especialmente com o fato de que os profissionais que demonstravam pouca aceitação e sentimentos negativos (medo, insegurança e falta de identificação) quanto à internação psiquiátrica no hospital geral, foram justamente aqueles que não trabalharam anteriormente com tais sujeitos ${ }^{(7-8)}$. Assim, é fundamental que os profissionais de enfermagem valorizem os aspectos psíquicos dos indivíduos internados nestas instituições, o que pode ser facilitado por meio de ações educativas.

Entretanto, a pouca afinidade apresentada por parte da equipe de enfermagem em relação à natureza psíquica do indivíduo internado não impediu a realização de procedimentos, uma vez que, mesmo inseguros, estes profissionais ofereciam a mesma assistência clínica que era concedida às demais pessoas hospitalizadas.

No que se refere às estratégias terapêuticas no campo da psiquiatria, nota-se que, gradualmente, está ocorrendo mudança no entendimento quanto à prestação da assistência e às formas de tratamento dos transtornos mentais, pois o tratamento medicamentoso não é mais visto como método único e exclusivo e sim mais um recurso terapêutico aliado a outros, tais como a formação de vínculo com as pessoas hospitalizadas ${ }^{(19)}$. Recentemente, estudos relatam que os enfermeiros que atuam na área psiquiátrica devem realizar a assistência baseados nas características particulares de cada sujeito que estão cuidando(18).

Em relação a estas estratégias, notou-se que alguns profissionais que participaram desta investigação utilizaram o relacionamento terapêutico. Para que a assistência de enfermagem em saúde mental possa ser eficaz, é importante que os profissionais procurem oferecer mais atenção, diálogo e demonstrar interesse diante dos pensamentos, sentimentos e comportamentos dos pacientes ${ }^{(19)}$.

Nesta pesquisa, notou-se que alguns profissionais dispunham de ferramentas como estabelecer confiança e diálogo com os pacientes, para que assim a assistência pudesse ser realizada sem intercorrências (como tentativas de agressão contra si ou terceiros, danos a dispositivos e utensílios do hospital, ou até mesmo a evasão da instituição). Para se alcançar essa eficácia, indica-se a utilização da comunicação terapêutica durante todo o período de internação psiquiátrica, e não apenas em momentos de intervenções psicoterapêuticas ${ }^{(20)}$.

Por fim, é possível inferir que os sentimentos da equipe de Enfermagem frente à pessoa com transtorno mental internada em hospitais gerais são, em geral, semelhantes entre muitos profissionais. Nessa perspectiva, é preciso centrar a atenção para aqueles que não demonstram estar adequadamente treinados e não apresentam mudanças de atitudes e pensamentos no que se refere à assistência a estas pessoas, para que assim seja oferecido cuidado humanizado, integral e sem discriminação por parte da equipe. Nesse sentido, pode ser considerada uma limitação do estudo a análise dos profissionais de enfermagem de forma geral, sem focarmos as especificidades inerentes às atuações de técnicos e enfermeiros no cuidado às pessoas hospitalizadas. E o fato de ser recente (desde dezembro de 2014) a internação psiquiátrica no hospital que constituiu cenário deste estudo, também pode ser considerada uma limitação.

\section{CONCLUSÃO}


Evidenciou-se uma equipe resistente e com sentimentos fundamentados no estigma que a doença mental carrega ao longo dos tempos. Observa-se também que a capacitação oferecida por este serviço de saúde à equipe de enfermagem não foi suficiente diante das necessidades psíquicas da pessoa com transtorno mental.

Assim, mesmo identificando que um dos grandes avanços do movimento de reforma psiquiátrica foi a implantação de leitos em hospitais gerais, tais como estes que constituíram o cenário desta investigação, os dados obtidos nesta pesquisa sinalizam limites e desafios a serem superados.

Um dos grandes desafios é a qualificação para assistência digna. Nesse sentido, destacam-se as seguintes necessidades: realização de intervenções educativas que abordem a cidadania da pessoa com transtorno mental, assegurada pelo direito à igualdade; implantação de encontros/ reuniões interdisciplinares para a discussão de casos clínicos e elaboração de um plano terapêutico individualizado, no qual a equipe de enfermagem possa direcionar os cuidados para as necessidades psíquicas, bem como agregar habilidades relacionais às habilidades técnicas inerentes à profissão.

\section{REFERÊNCIAS}

1. Ribeiro JCS, Dacal MPO. A instituição hospitalar e as práticas psicológicas no contexto da Saúde Pública: notas para reflexão. Rev. SBPH. [Internet] 2012;15(2) [acesso em 24 fev 2016]. Disponível: http://pepsic.bvsalud.org/ scielo.php?script=sci_arttext\&pid=S1516-08582012000200006.

2. Mosimann LTQ, Lustosa MA. A Psicologia hospitalar e o hospital. Rev. SBPH. [Internet] 2011;14(1)[acesso em 24 fev 2016]. Disponível: http://pepsic.bvsalud.org/scielo.php?script=sci_arttext\&pid=S1516-08582011000100012.

3. Lopes AC. Tratado de Clínica Médica. São Paulo: Roca; 2009.

4. Paes MR, Silva TL, Chaves MMN, Maftum MA. O papel do hospital geral na rede de atenção à saúde mental no Brasil. Cienc Cuid Saude. [Internet] 2013;12(2) [acesso em 24 fev 2016]. Disponível: http://dx.doi.org/10.4025/ cienccuidsaude.v12i2.14207.

5. Ministério da Saúde (BR). Portaria n. 148, de 31 de janeiro de 2012. Define as normas de funcionamento e habilitação do Serviço Hospitalar de Referência para atenção a pessoas com sofrimento ou transtorno mental e com necessidades de saúde decorrentes do uso de álcool, crack e outras drogas. [Internet] Brasília: Ministério da Saúde; 2012 [acesso em 24 fev 2016]. Disponível: http://portalsaude.saude.gov.br/images/pdf/2015/marco/10/ PORTARIA-148-31-JANEIRO-2012.pdf.

6. Ferreira GMK, Marins MF, Moraes RS, Lopes CLAS. Internações psiquiátricas em hospital geral e a política de saúde mental. J Nurs Health. [Internet] 2012;2(Suppl) [acesso em 24 fev 2016]. Disponível: https://periodicos. ufpel.edu.br/ojs2/index.php/enfermagem/article/view/3477/2862.

7. Silva NG, de Oliveira AGB. Interconsulta psiquiátrica e unidades de internação psiquiátrica no Brasil: uma pesquisa bibliográfica. O Mundo da Saúde. [Internet] 2010;34(2) [acesso em 24 fev 2016]. Disponível:http://www. saocamilo-sp.br/pdf/mundo_saude/75/244a251.pdf.

8. Silva NG, Silva PP, de Oliveira AGB. A percepção dos trabalhadores de Enfermagem sobre a assistência à saúde mental em hospital universitário. Cienc Cuid Saúde. [Internet] 2012;11(2) [acesso em 24 fev 2016]. Disponível: http://dx.doi.org/10.4025/cienccuidsaude.v11i2.11181.

9. Schmitt R, Gomes RH. Aspectos da interconsulta psiquiátrica em hospital de trauma. Rev. psiquiatr. Rio Gd. Sul. [Internet] 2005;27(1) [acesso em 24 fev 2016]. Disponível:http://dx.doi.org/10.1590/S0101-81082005000100009.

10. Bardin L. Análise de conteúdo. Lisboa: Edições 70; 1995.

11. Braun V, Clarke V. Using thematic analysis in psychology. Qual Resin Psychol. [Internet] 2006;3(2) [acesso em 24 fev 2016]. Disponível: http://eprints.uwe.ac.uk/11735/2/thematic_analysis_revised.

12. de Lima DU, Garcia APRF, Toledo VP. Compreendendo a equipe de Enfermagem na assistência ao paciente esquizofrênico. Rev Rene. [Internet] 2013;14(3) [acesso em 24 fev 2016]. Disponível: http://dx.doi.org/10.15253/ rev\%20rene.v14i3.3421. 
13. Oliveira LRM, de Carvalho CD, de Carvalho CMS, da Silva Júnior FJG. O ensino da saúde mental para Enfermagem: uma revisão da literatura. R. Interd. [Internet] 2013;6(2) [acesso em 24 fev 2016]. Disponível: http:// revistainterdisciplinar.uninovafapi.edu.br/index.php/revinter/article/view/60.

14. Jorge-Monteiro F, Madeira T. Considerações sobre doença mental e comunicação social.Anál. psicol [Internet] 2007;1(25) [acesso em 24 fev 2016]. Disponível:http://www.scielo.mec.pt/pdf/aps/v25n1/v25n1a08.pdf.

15. Marcon SR, Conciani ME, de Oliveira JRT. Qualidade de vida entre trabalhadores de Enfermagem de um hospital universitário. Espaç. Saúde (Online). [Internet] 2014;15(1) [acesso em 24 fev 2016]. Disponível: http:// dx.doi.org/10.22421/1517-7130.2014v15n1p6.

16. Camuccio CA, Chambers M, Välimäki M, Farro D, Zanotti R. Managing distressed and disturbed patients: the thoughts and feelings experienced by Italian nurses.J Psychiatr Ment Health Nurs. 2012;19(9):807-15.

17. de Lima RVM, Pedrão LJJ, Miasso AI, da Costa Júnior ML. Papéis, conflitos e gratificações de enfermeiros especialistas em Enfermagem psiquiátrica e saúde mental. Rev. Eletr. Enf. [Internet] 2012;14(1) [acesso em 24 fev 2016]. Disponível: http://dx.doi.org/10.5216/ree.v14i1.12145.

18. Amorim AMMNE, Cruz DKRC, Cardoso MLLO. Percepção do enfermeiro no cuidar ao doente mental: uma revisão de literatura. Rev. Multip. Saúde HSM [Internet] 2013;1(2) [acesso em 24 fev 2016]. Disponível:http://ojs. saomarcos.org.br/ojs/index.php/cientifica/article/view/2/20.

19. Guimarães AN, Borba LO, Maftum MA, Larocca LM, Nimtz MA. Mudanças na atenção à saúde mental decorrentes da reforma psiquiátrica: percepções dos profissionais de Enfermagem. Cienc Cuid Saúde. [Internet] 2015;14(1) [acesso em 24 fev 2016]. Disponível: http://dx.doi.org/10.4025/cienccuidsaude.v14i1.22187.

20. Coelho MTV, Sequeira C. Comunicação terapêutica em Enfermagem: como a caracterizam os enfermeiros. Rev. port. enferm. saúde mental.[Internet] 2014;(11) [acesso em 24 fev 2016]. Disponível: http://www.scielo.mec. pt/scielo.php?script=sci_arttext\&pid=S1647-21602014000200005. 